\title{
Cooperation, Interaction, Search: Computational approaches to the psychology of asking and answering questions
}

\author{
Christina Boyce-Jacino* \\ Simon DeDeo ${ }^{\dagger}$
}

\begin{abstract}
This chapter argues that question-asking should be studied as a process of interactive search between social agents, and shows how new tools in machine learning, combined with large-scale data sets on question-asking "in the wild", can give us new insights into how it works beyond the confines of the laboratory. We introduce the technical aspects of these tools, new mathematical frameworks that can use them to gain insights into psychology of asking and answering questions, an examples of their application to the online forums Stack Exchange and Mumsnet, and archives of crosswords and quiz questions. We draw attention to the accompanying challenges, including the difficulty of using metadata as dependent variables, and determining the construct validity of the tools themselves.
\end{abstract}

One of the most common ways we make sense of the world is by asking questions. Understanding how this works combines classic elements of psychology with social science questions about how question-asking emerges in the context of shared norms and expectations. This chapter will show how advances in natural language processing and the emergence of new, large-scale datasets, combined with the development of new conceptual and mathematical frameworks to organize their outputs, give us new ways to look at this problem.

While "questions" in the purely linguistic sense may serve a pragmatic function of continuing a conversation, making a point (Searle, 1969), or as part of a pedagogical practice (Yu, Bonawitz, \& Shafto, 2019), the basic task is one of cooperative, interactive search. Over and above its intrinsic interest, these three features of question-asking make it particularly intriguing for psychologists.

First, question-asking is cooperative: the basic task involves a common goal where the person asking and the person answering collaborate, to some extent, on increasing the sum of their knowledge. One can not answer a question that someone else has not, in some fashion, participated in asking. It is tempting to strengthen this and to say that, in the "ordinary" case, there is usually a great deal of common knowledge about whether or not participants

\footnotetext{
*Department of Social and Decision Sciences, Carnegie Mellon University, Pittsburgh, PA 15213

${ }^{\dagger}$ Department of Social and Decision Sciences, Carnegie Mellon University, Pittsburgh, PA 15213; Santa Fe Institute, 1399 Hyde Park Road, Santa Fe, NM 87501
} 
are engaged in a question-asking task, and the norms of that engagement. It is also tempting to focus on the knowledge of the asker, and to understand the task primarily in terms of increasing their state of knowledge. However, question-asking in the real world is rarely so simple. One of the challenges in the data science era, we find, is that participants in a question-asking task may have a great deal of disagreement about what, exactly, is going on; they may be mistaken about who has more to learn; and much of what is, finally, learned, may not be intended or desired.

Second, question-asking is interactive: the participants must not only exchange information, but adjust the information that they exchange in response to what they receive from the other. In the simplest cases, the answerer uses features of the question to determine the information required, and possibly the asker's background knowledge, but many questionasking tasks in the real world, as we shall see, involve a dialogue where asker and answerer refine their information about the both the matter at hand and the abilities, needs, and intentions of the other person.

Third, question-asking is a form of search, and as such, exploratory: the participants are, at least to some extent (and neglecting edge cases such as the ritual of a catechism), uncertain about the final outcome of the engagement. This uncertainty is usually a defining feature of the interaction, often (though not always) because of the question-asker's uncertainty. The uncertainty may be "simple", and restricted a finite, pre-stated range of possibilities (e.g., which of three buses go in the correct direction), or open-ended (e.g., how to solve a mathematical problem, where the questioner "doesn't know where to begin"); it may also be unexpected (e.g., the questioner asks "is it A or B?", but the answer is "C".)

To understand the cognitive foundations of question asking, researchers have traditionally turned to laboratory investigation. Careful experimental work yielded several formal models by which questions are evaluated and selected (Rothe, Lake, \& Gureckis, 2016, 2017; Nelson, 2005; Cohen \& Lake, 2016). A key advantage of these formal frameworks is that they connect to normative prescriptions of how people should ask questions. A disadvantage, however, is that they are challenging to apply to the rich variety of questions asked and answered in real world situations. What laboratory experiments gain in experimental control, they sacrifice in ecological validity.

In the past decade, and in part simply due to the ubiquity of the activity, question-asking has also drawn the attention of those in machine learning. Advances in these domains have been powerful, but have primarily been driven by access to large, and focused training data sets. Consequently, they are limited in their ability to generalize to inquiry behaviors outside of a specific domain or context (Mostafazadeh et al., 2016; Wang \& Lake, 2019; Chali \& Hasan, 2015).

We begin this chapter by grounding question-asking in basic psychology. We follow with a series of demonstrations of how advances from machine learning and natural language processing (NLP), applied to real-world data sets, can help us tease apart the social and psychological demands of the task and overcome the limitations of laboratory work. We address the significant challenges faced by studying question-asking at "at scale" in realworld conditions, and the promising implications for combining data science, laboratory work, and mathematical theory. 


\section{The Psychology of Question-Asking}

The simplest kinds of questions - from a psychological standpoint - are those posed not to other people, but to databases or to "nature itself", understood as static "oracles" bound to give literal and accurate answers. This minimally-interactive setting isolates the exploratory feature of question asking, which is cashed out in a Bayesian framework with the mathematical formalism of Optimal Experiment Design (OED; Rothe et al., 2017; Coenen et al., 2019).

Within this framework, a questioner begins with a goal (something they wish to learn) and a space of possible questions she could pose. She then evaluates that set according to how valuable she expects answers would be given her current state of knowledge; the question ultimately selected is the one which has the greatest expected reduction in uncertainty about a hypothesis, with a standard metric for a question being the Kullback-Leibler divergence from the current state of knowledge, to the state of knowledge after receiving an answer to that question, averaged over the probabilities of the different answers (given the current state of knowledge).

OED focuses on the "search" value of a question. It provides a clear and informationtheoretically justified baseline for predicting the questions that people will as ( or, at least, quantifies their deviations from a normative baseline). The vast majority of queries, however, are not posed to oracles, but unfold in social contexts where cooperation and interactivity come to the fore. The search for information, rather than being individual and private, occurs in the setting of dialogue and as such requires a deeply intertwined process of communicating and inferring goals, intentions, and knowledge - features that violate the underlying assumptions of the OED framework (Hawkins \& Goodman, 2020).

Cooperation and interaction greatly may complicate the study of question-asking, but they also make it possible. We are remarkably good at inferring the goals of others given their actions and gestures (Moore et al., 2015; Tomasello et al., 1997), inferring the intent of objects given their movement (e.g., Scholl \& Gao, 2013), and inferring the intentions of others from their speech. In each of these domains, we are able to observe an action and from that, reason about the hidden mind of the actor.

Central to social behavior specifically is Theory of Mind (ToM); the suite of cognitive mechanisms underlying the capacity to reason about, and represent, the mental states of others (Baron-Cohen, 1991; Leslie, 1987). At a young age children are able to explain a person's action in terms of their hidden mental states (Onishi \& Baillargeon, 2005). For instance, in a false belief task, a canonical demonstration of ToM, infants and children must recognize that the beliefs held by an actor differ from both their own personal beliefs, and the true state of the world (Wimmer \& Perner, 1983; Perner, 1991; Onishi \& Baillargeon, 2005). Representing an actor's mental states in this way requires a child to hold a representation of that other person's mind, and use that representation to reason about how that person is likely to act. This capacity has been formalized as a Bayesian problem of inverse planning(Ullman et al., 2009; Tenenbaum et al., 2006).

Mentalization is crucial to both the cooperative and interactive aspects of questionasking. A questioner may have a specific goal about acquiring a piece of information about the world, and while an answerer may have what is needed, he will need to infer that goal on the basis of the query alone. At a second-order level, a question-asker who understands 
this complexity should pose her question in a way to make that task easier, and at a higher order yet, an answerer might endeavor to convey that he knows this attempt has been made.

Casting question asking and answering as an intertwined process of inference sets the stage for building a framework of inquiry as one in which representations of knowledge and goals are shared and changed through linguistic dialogue.

The examples detailed below span large data sets of text from online discussion forums, question-answer sites, crossword puzzles, and quiz shows. They show, first, how new approaches provide a richer notion of the "search" implicit in question-asking, and then, second, how they can give us new handles on its cooperative and interactive aspects.

\section{Search: From Lexicons to Meanings}

Much of the methodological advances made possible in the data science era are based on a very simple insight: in successful dialogues, speakers tend to borrow each other's meanings. This correspondence happens on multiple levels, broadly categorized as semantic, syntactic, and lexical (Levelt \& Kelter, 1982; Garrod \& Anderson, 1987; Garrod \& Clark, 1993; Branigan et al., 2000). In the case of semantic coordination, participants in a conversation converge to a shared mental model and representation of relevant information.

In an early demonstration of this convergence, Garrod and Anderson (1987) showed that pairs of participants tasked with solving a maze converged on particular types of descriptions associated with mental models of the task (e.g., in terms of paths among points, or moves on a coordinate system). Lexical coordination emerges when two partners use the same expressions to reference their shared representations, and syntactical coordination when they use the same grammatical structures (Branigan et al., 2000).

When we look at question-asking in the wild, it is easy to find strong signals of the basic effect. This is shown in Fig. 1 for a large database of question and answer pairs from the website "Stack Exchange" 1 an online forum where a community of participants post questions on a variety of topics, provide answers, and engage in follow-up queries. Two example forums were used here: questions and answers from the Biology forum, and from the Bitcoin forum. When questions are matched with their answers, the median proportion of shared words is around $11 \%$. When questions are matched instead with random answers in the same forum, this lexical overlap drops (median overlap is 5.6\%), and it drops further when questions are matched with random answers from a different forum entirely (median overlap across topics is $0.33 \%$ ).

The accompanying table presents the percentage of question-answer pairs which share at least one word. As expected, in nearly $100 \%$ of cases, when questions are matched with their proper answers, they share words; conversely only about $50 \%$ of random pairings share words. This difference becomes more stark when words are filtered according to frequency of occurrence in common useage. Now, $80 \%$ of matched pairs share words, while almost no randomly matched pairs have words in common. This suggests that answers are particularly well situated to their prompting questions; they share infrequent words.

That these signals are strong - effects at the level of orders of magnitude - is happy, but not, of course, surprising. Most obviously, strong lexical overlap is due to the way in

\footnotetext{
$1_{\text {https://biology.stackexchange.com }}$ and https://bitcoin.stackexchange.com
} 


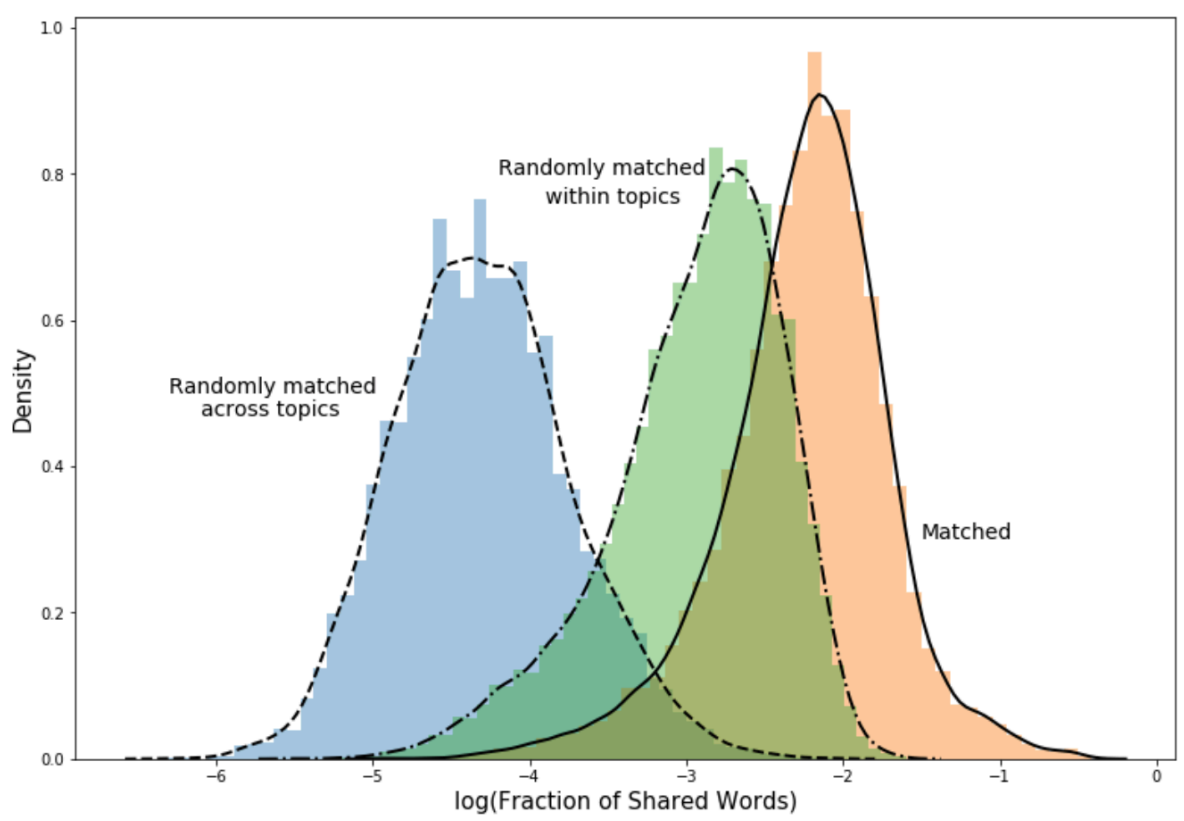

All words Only infrequent words

\begin{tabular}{lll}
\hline Matched, within Biology & $99.6 \%$ & $80 \%$ \\
Random pairs, within Biology & $96.5 \%$ & $6.2 \%$ \\
Randomly pairs, Biology/BitCoin & $50.5 \%$ & $0.02 \%$ \\
\hline
\end{tabular}

Figure 1: Lexical coordination in online question-asking. Top: the distribution of overlap (fraction of shared words between question and answer, conditional upon at least one match) for question and answer pairs in the Stack Exchange Biology forum (orange), compared to a null model where questions and answers are paired randomly within the forum (green), and randomly between the biology forum and the BitCoin forum. Bottom: how overlap varies when thresholded by frequency (either, all words, or only words that appear less than once per 100,000 in general use. 
which the question initiates a search process: a (reasonably good) question will (often) use terminology that is roughly in the ballpark of where the answer is expected to be, enabling the subsequent search to be more efficient. Meanwhile, we expect that a (reasonably good) answer will naturally share some of the words found in the question, simply by virtue of being on topic. An answer to a question about the use of genetic data for validating a taxonomy of bird species is expected to use words like "genetic" and "bird", that also appear in the question.

The second effect is more subtle, and due to the cooperative and interactive aspects of question-asking. Even when the question is completely mistaken about the correct domain, the person providing the answer may wish to communicate, for example, that she understands the question-asker's beliefs about where the answer is likely to be found - even if only to follow up with an explanation as to why those beliefs are incorrect. If, for example, genetic data is never used to validate an avian taxonomy, a good answer to the question "how are avian taxonomies established, e.g., by genetic data?" may still refer to that (mistaken) belief by using the word "genetic".

Teasing apart these effects is made easier by NLP tools that map words into a latent, high dimensional, "semantic" space. By going beyond the word level, we can look for the effects of not just shared lexicon, but also - in some sense - shared meaning. This allows us to capture borrowing that occurs at the level of meaning, such as a question about "bird genetics" that is answered by reference to "avian DNA".

The class of models used to represent words in this way are known as embedding algorithms. They are founded on the hypothesis that words that appear together and in similar contexts are likely to be semantically related (Baroni et al., 2014; Firth, 1957; Harris, 1954). Embedding models are often constructed by machine learning methods in the following way. First, the engineer trains an algorithm to predict the word that comes next in a sentence, on the basis of the words that have come before, using a very large database of sentences. These algorithms take words as input, and as part of the training process simultaneously construct (1) a way to represent each word as a vector of numbers (the "internal" representation), and (2) a way to use that vector to predict the words that come next.

It turns out that the internal representations capture something about the semantics of the corresponding words, at least in a relational sense: words whose internal representations are mathematically similar are also similar in terms of their "meaning". These representations are naturally interpreted geometrically: words with similar meanings are placed nearby in (a high-dimensional) space.

While many embedding algorithms have been developed (see GloVe by Pennington, Socher, \& Manning, 2014, FastText Bojanowski, Grave, Joulin, \& Mikolov, 2016, and below), the examples presented in this section use word2vec (Mikolov et al., 2013). A shallow neural network consisting of one hidden layer, a "SkipGram" word2vec takes a target word as input; say, "dog" from the utterance "Is my dog the cutest pet?". Given the target word "dog", the task of word2vec is to predict the neighboring words "is," "my," "the," "cutest," and "pet." The internal representation yields a vector of features which reflect how the word "dog" is used in language.

The promise of these models is that they encode realistic semantic representations. A wealth of literature has emerged to support the veracity of that claim (see, e.g., neural mapping evidence from Pereira et al., 2018; evidence from classic psycholinguistic tasks 

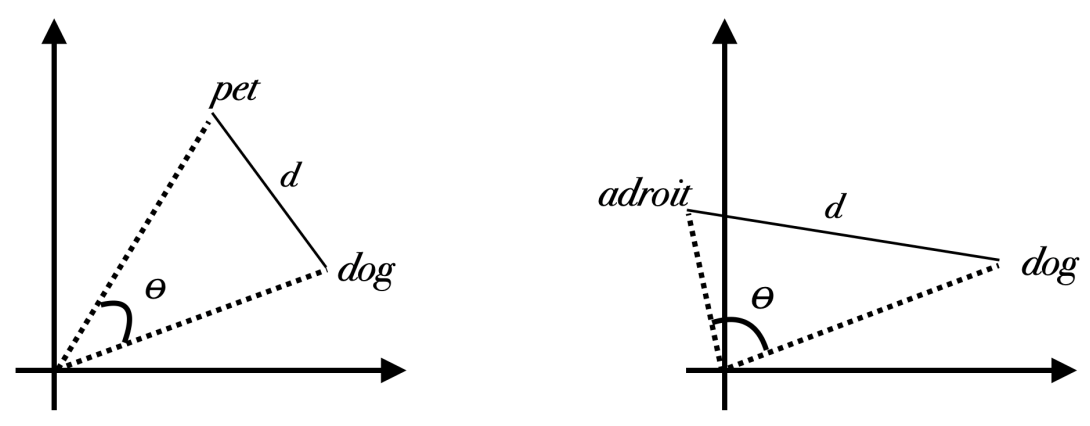

Figure 2: An example of how words relate to each other in a vector space, where $\theta$ represents the cosine distance between two words, and $d$ represents Euclidean distance. Words that are often used together in language, such as pet and dog, have a smaller cosine distance and Euclidean distance $(\theta=44.2, d=2.2)$ than two words which are used in vastly different contexts such as adroit and $\operatorname{dog}(\theta=90.8, d=4.4)$.

in Mandera, Keuleers, \& Brysbaert, 2017; cognitive plausibility of distributional semantic models in general in Steyvers \& Tenenbaum, 2005). This remains an open question, and we return to this topic below.

For any vector space, there are many different notions of distance. One is cosine distance: a measure of how close two vectors are based on the angle between them. Words which are semantically similar will have a cosine distance close to zero, whereas semantically orthogonal words will have similarities approaching 90 degrees. The example pictured in Fig.2 presents the case in which the word "dog" sits close to "pet" but nearly ninety degrees from "adroit," with cosine distance labeled $\theta$. These relationships are intuitive: while dog and pet are likely to be used in similar situations regarding furry companions, an adjective such as adroit is unlikely to share this context. Another distance measure is Euclidean, or "straight line" distance, labelled $d$ in the figure. Models of this kind have been shown to capture features relevant to decision-making processes, such as similarity among items in a choice set (Bhatia, 2019) and consumer purchasing behavior (Bhatia \& Olivola, 2018), as well as social psychological features of stereotyping (Lewis \& Lupyan, 2019).

Practically speaking, measures of semantic similarity are often used as independent variables to predict some feature of interest in the question-asking task. We gain insight into the psychology of question-asking by looking at how the cognitive aspects of the cooperative, interactive, navigation of semantic space predict relevant outcomes.

An example of how this can be done to study question-asking is provided by our recent work on how the semantic relationship between a question and its (correct) answer affect difficulty (Boyce-Jacino \& DeDeo, 2020). There, we proposed that questions are made difficult by changes along two abstract dimensions: obscurity, or the rarity of its answer words in ordinary use, and opacity, the indirectness of the question cues in semantic space.

Each of these dimensions are grounded in psychological intuitions. From a Bayesian perspective, a question with an obscure answer will be difficult because of the low prior on the words. A greater amount of evidence needs to accumulate before that answer is selected 
A

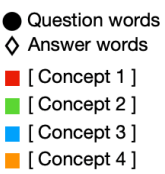

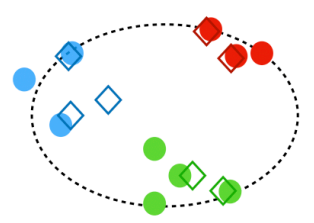

B

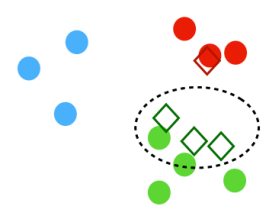

C

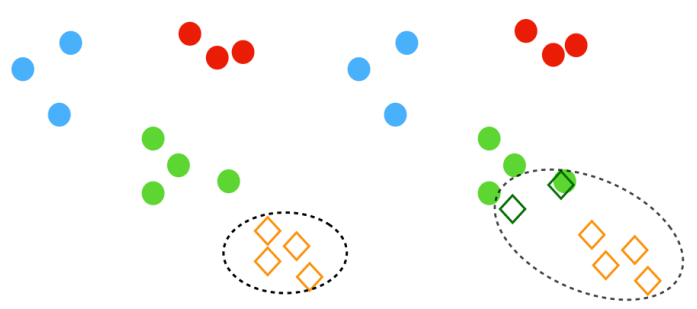

Figure 3: A "cartoon" illustration of the high-level semantic spaces occupied by questions (circles) and answers (diamonds). To highlight the relationship between the answer concepts and question concepts, the dotted line represents the coverage of the answer.

than if the answer was common and therefore expected (Oaksford, Chater, et al., 2007). The role of opacity, meanwhile, suggests that the cognitive process of answering also plays a role; regardless of the rarity of an answer, if the semantic cues within the question do not point to it, navigating to the correct conceptual space will be difficult.

The theory is validated with an analysis of 24 years of New York Times crossword puzzles, and 33 years of questions from the quiz show Jeopardy. Importantly, questions in these data sets come tagged with measures of difficulty. Both obscurity, operationalized with word frequency in common speech, and opacity, operationalized using cosine distance (in word2vec space) between words in the question and the answer, increase with answer difficulty.

One can think of opacity and obscurity as decomposing two distinct pieces of a question's "search value" hidden within the OED framework described above. What this work shows is how an answer can be unexpected because it is rarely given (obscure), or because, despite being rather common in general, it is not strongly semantically connected with questions of that type (opaque). Both matter, although, somewhat satisfyingly, crossword puzzles tend to increase difficulty by varying opacity, while Jeopardy questions rely more on obscurity.

Identifying opacity and obscurity as independent contributors to difficulty can help us understand the ways in which people try to overcome them socially, for example, or the underlying memory-retrieval and navigation mechanisms that are affected by variation along the two axes. One underlying assumption of this work is that word embeddings generated by vector space models correspond, in some way, to how semantic information is organized in memory, and that the mathematical properties of vector spaces reveal how that organization is traversed, a question we return to below.

\section{Cooperation and Interaction in Semantic Space}

The spatial metaphors of vector-space representations give us new ways to think about the question-asking process. Fig. 3 shows an example of how the words in a question (filled circles) might spread out over a semantic space, and how the words in an answer (open diamonds) might relate. In case $\mathrm{A}$, for example, the answer overlaps all of the conceptual groupings of the question while in case $\mathrm{B}$, it narrows down onto one of the groups. In case 
$\mathrm{C}$, the answer introduces an entirely new concept (the orange diamonds), while in case D the introduction of this new concept is accompanied by reference to one of the concepts in the original question.

Viewing question and answer pairs in this way allows us to consider effects beyond pure information transmission. Imagine, for example, that the correct answer to the question involves the semantics of Concept 2. Both A and B are now (potentially) valid answers from the point of view of information gain, but they have different cooperative and interactive properties. While Answer B, in this case, is "straightforward", Answer A, for example, may be more successful because it reassures the question-asker that the answerer has paid attention to other implicit suggestions of where the answer might lie. If the correct answer involves Concept 4, then Answer D may be more successful than Answer C for similar reasons.

Perhaps happily, adequate representations of our semantics usually involve much more than just two dimensions - word2vec, for example, places words in a three hundred dimensional space. This makes the relationships impossible to visualize in any simple fashion. Because of this, we present, in Table 1, five measures that attempt to capture the different aspects of the question-answer relationship, based on work presented in Boyce-Jacino and DeDeo (2019).

The first two features, radius and clustering, refer to semantic aspects of a question, or an answer, considered in isolation. Radius captures how much semantic space is covered by the text: when a question has high radius it includes many semantically distinct features. Clustering is a more subtle concept, and captures the extent to which this coverage is clumped in distinct locations.

An example of what clustering "looks like", from Boyce-Jacino and DeDeo (2019), is shown in Fig. 4. Both questions in the example are concerned with the problem of dietary restrictions, and have have similar radii. However, in the high clustering case, the question clusters around a multiple themes, including that of celebration ("birthday", "commemorate", etc) and the idea of trying ("striving", "attempting", etc).

The next three features capture dyadic relationships between question and answer. Narrowing captures the extent to which the answer is more (or less) focused than the question. In Fig. 3, for example, Answer A has a similar radius to the question, while Answers B, C and D have much smaller radii (and thus a high "narrowing"). Shift captures the extent to which the answer covers new material (e.g., Answer $\mathrm{C}$ has larger shift than answer D, and both have larger shifts than Answers A and B). Finally, focus captures the extent to which an answer matches some, but not all, of the question; for example, Answer B has higher focus than Answer A.

These measures have natural relationships to quantities in the framework of Bayesian cognition (Griffiths, Chater, Kemp, Perfors, \& Tenenbaum, 2010), and for this reason we refer to them as (conjectural) parts of a "Bayes cloud" decomposition. Radius can be thought of as a measure of uncertainty, in the information-theoretic sense; a high radius question is more uncertain than a low radius question. Clustering can be thought of as a measure of (internal) mutual information, or how much one part of the question predicts other parts. Shift is measures of surprise, or information gain, and focus a measure of mutual information between question and answer.

These features can then be used to investigate the interactive nature of question asking. 


\begin{tabular}{|c|c|c|}
\hline Feature & Operationalization & Definition \\
\hline \multicolumn{3}{|l|}{ Individual } \\
\hline Radius & $\begin{array}{l}\text { Average pairwise distance between words; } \\
\text { how "spread out" the text is over semantic } \\
\text { space }\end{array}$ & $R=\frac{1}{n(n-1)} \sum_{i \neq j}^{n} d\left(\vec{x}_{i}, \vec{x}_{j}\right)$ \\
\hline Clustering & $\begin{array}{l}\text { Variance in pairwise distance between } \\
\text { words; how "clumpy" the text is in seman- } \\
\text { tic space }\end{array}$ & $\frac{1}{n(n-1)} \sum_{i \neq j}^{n}\left(d\left(\vec{x}_{i}, \vec{x}_{j}\right)-R\right)^{2}$ \\
\hline \multicolumn{3}{|l|}{ Dyadic } \\
\hline Narrowing & $\begin{array}{l}\text { The (log) ratio of the radius of question } \\
\text { and answer; how much the answer narrows } \\
\text { down on some part of space }\end{array}$ & $\log \left(\frac{R_{Q}}{R_{A}}\right)$ \\
\hline Shift & $\begin{array}{l}\text { Average pairwise distance between ques- } \\
\text { tion and answer words; how much the an- } \\
\text { swer moves away from the material in the } \\
\text { question }\end{array}$ & $S=\frac{1}{n_{A} n_{Q}} \sum_{i, j}^{n_{A}, n_{Q}} d\left(\vec{x}_{i}^{A}, \vec{x}_{j}^{Q}\right)$ \\
\hline Focus & $\begin{array}{l}\text { Variance in pairwise distance between } \\
\text { question and answer words; how much the } \\
\text { answer focuses on some parts of the ques- } \\
\text { tion compared to others }\end{array}$ & $\frac{1}{n_{A} n_{Q}} \sum_{i, j}^{n_{A}, n_{Q}}\left(d\left(\vec{x}_{i}^{A}, \vec{x}_{j}^{Q}\right)-S\right)^{2}$ \\
\hline
\end{tabular}

Table 1: Elements of the "Bayes cloud" decomposition of questions and answers in semantic spaces. Here $\vec{x}_{i}$ refers to the semantic vector representation of word $i$ in a text, and $n$ the number of words in the text, with superscript A or Q indicating whether it is the question or the answer in the dyadic cases; $d(\vec{x}, \vec{y})$ refers to a distance measure (which could be, e.g., Euclidean or cosine distance) between semantic vectors $x$ and $y$. 


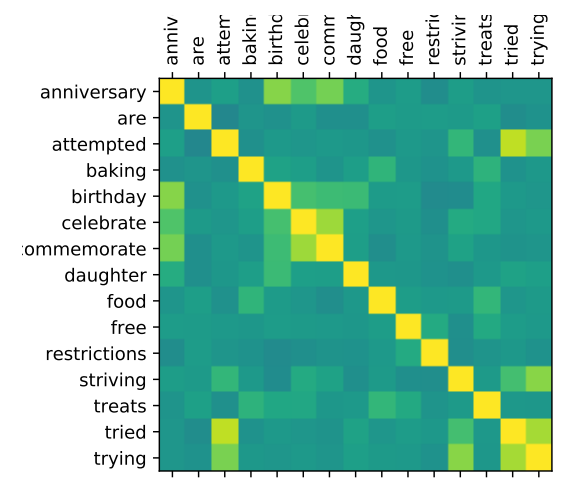

(a) High clustering question

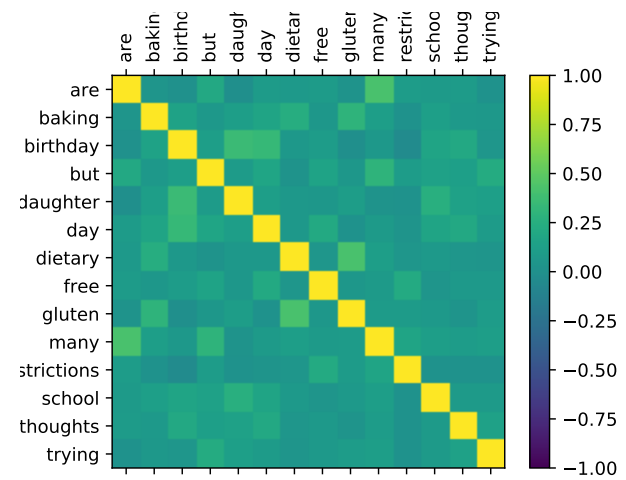

(b) Low clustering question

Figure 4: An example of high- and low-clustering questions in Mumsnet, with similar topics and question length. Pairwise cosine similarity is plotted for each questions; the shading reflects the strength of semantic similarity between two words such that highly related words are lighter in color (yellow) than minimally related, or orthogonal words (dark green). In (a), the query has multiple areas where semantic information is concentrated (e.g., a "birthday" cluster, a "food" cluster, and a "striving/attempting/trying" cluster). In contrast, the information in (b) is more uniform; none of the words are particularly related to one another.

In particular, Boyce-Jacino and DeDeo (2019) used a dataset of threads from the online parenting website, Mumsnet. $!^{2}$ where parents can ask, and answer, questions relevant to parenting. We can regress the features defined in Table 1 on, in particular, a proxy of answer quality: whether or not a question asker returns to the thread after posting their query. The assumption here is that if the original question asker has to return to the thread after observing the answers, the given answers are likely unsatisfactory in some fashion 3

Two simple predictors of whether or not the question-asker returns to the thread is length of question and length of answer. As the length of prompting question increases, the probability that the poster will return to the thread decreases. Conversely, longer answers are more likely to prompt questioner return than ones with greater brevity.

Semantic features of the question-answer pair, however, appear just as strongly. Answer radius (but not question radius), for example, plays an important role: answers with larger radii are more likely to lead to asker return, suggesting an effect related to how well the question-asker was able to, initially, narrow down the search space. Clustering also has a strong effect, in the opposite direction: the likelihood that the asker returns decreases as either question or answer become more clustered. If you wish to receive a satisfying answer, in other words, it helps if your question is structured into smaller units, and similarly for the answer you hope to receive in turn.

Shift and focus also matter. An answer with higher shift increases the probability of re-

\footnotetext{
${ }^{2}$ https://www.mumsnet.com

${ }^{3}$ Some parts of Mumsnet are more discussion oriented, where the return of the original poster may be considered a positive event; we focus on the "problem-solving" parts of the site explicitly oriented towards question-answering, and drop the cases where an original poster returns simply to say "thank you" to the people who answered.
} 
turn, while higher focus decreases it. If you are introducing new information to the questionasker (quantified by shift), it helps to include some material from groups included in the question itself (quantified by focus). This is the difference between Answer C and Answer D in our cartoon Fig. 3 .

These effects go beyond the standard conceptualization of question-asking as information seeking. If the previous section used data science to tease apart different aspects of the "search" aspect of question-asking, the features of Table 1 give us new handles on its cooperative and interactive features.

In each of these cases, the strength of the effect (in normalized $z$-score units) depends upon the definition of distance used, and the best notion of distance-i.e., the one most closely matching underlying cognitive mechanisms - remains an open question. For instance, recent work by Nickel and Kiela (2017) demonstrates that hyperbolic spaces far outperform Euclidean spaces in tasks that require the organization of information with strong inherent hierarchy, such as nouns.

\section{Better Semantic Models?}

Word2vec and related models provide information about the semantics of words taken in isolation: it will output a single vector for each word. This means that the vector for the word "mop" will be the same whether the word occurs in the sentences "mop up the floor" or "mop of hair" even though the word itself clearly means (to the human reader, at least) two different things.

A new line of work has emerged which seeks to address this problem by learning representations not at the level of words but at the level of word instances. If someone were to write the sentence "please ask the kid with the mop of hair to mop the floor" this new type of contextualized embedding model would generate separate word embeddings for each meaning of the word "mop".

A recent milestone for this was the development of BERT (Bidirectional Encoder Representations from Transformers; Devlin, Chang, Lee, \& Toutanova, 2018). BERT uses a transformer encoder (Vaswani et al., 2017), to create vector representations of words that are dynamically informed by the context in which they appear. One of the key features of the transformer architecture on which BERT runs is a self-attention mechanism which allows positional dependencies to be represented. Because it is bi-directional, it can represent positions and learn context not only from a forward reading of the text (left-right) but also from right-to-left. Unlike models such as word2vec, the way in which BERT is trained is not limited to predicting the next word (or words) in a sentence. Instead, it is trained first to predict a set of "masked" words, and then, to predict the next sentence in a sequence of sentences.

The resulting language representations can be used in whatever downstream tasks require vectorization of text. It is simple to generate examples, like the "mop" one above, where BERT performs extraordinarily well and can correctly place "mop" in "mop of hair" closer to words about haircuts than to words about brooms and vacuum cleaners. Recent research suggests, however, that BERT embeddings may not always offer significant improvements in more general tasks. Rather, BERT excels on tasks which hinge on a type of judgment where 
word order and syntax are important.

In one demonstration of this, Tenney et al. (2019) design a task to probe the nature of information encoded in a contextualized word embedding. Their "edge-probing" tasks focus on assessing how well baseline embedding models (which, like word2vec only have local information) and contextual embeddings encode higher level semantic information, syntactical structure, and long-range dependencies. They find that the benefits of contextual information lie primarily in the ability to represent syntax; BERT outperforms baseline models in constituent labeling tasks (which require knowledge of sentence structure), but does not offer much improvement on semantic tasks such as co-reference (a task of deciding whether two phrases refer to the same entity). Learning the surface patterns of language may be more difficult that learning compositional semantics.

That said, NLP models using BERT representations have shown a remarkable ability to capture complex issues in language, such as identification of hate speech (Kennedy, Jin, Davani, Dehghani, \& Ren, 2020), irony and sarcasm (Potamias, Siolas, \& Stafylopatis, 2019), and sentiment analysis of online product reviews ( $\mathrm{Xu}, \mathrm{Liu}, \mathrm{Shu}, \& \mathrm{Yu}, 2019)$. Many recent question-asking and answering architectures with contextualized embeddings as their foundational representations are successful in answering reading comprehension questions (e.g., Alberti et al., 2019; Wang et al., 2019).

The general structure of these models is to match a query with a passage of text to extract an "answer" from that passage (often, the focus is on modeling performance on the Stanford Question Answering Dataset compiled by Rajpurkar, Zhang, Lopyrev, and Liang (2016)). While performance tests on extant comprehension Q-A architectures give impressive results, the extent to which BERT represents and understands language itself remains unclear. From the point of view of the practicing psychologist, this may be the key goal: vector-space representations are better in as much as they map on to the conceptual structure of human users. A better understanding of exactly how BERT encodes context will help us judge whether it is a better tool than, say, word2vec for understanding human psychology.

\section{Challenges}

Laboratory experiments have so far dominated the study of question-asking. They have taught us a great deal about how queries are chosen and formed, how answers are integrated into knowledge, and how these abilities develop over time. While the promise of big data and computational methods are enormous, there are two primary strains of challenges to consider: the inherent messiness of big data, and the applicability of machine learning models to cognition.

When designing an experiment, a researcher has many tools at her disposal to deploy a controlled study. She constructs her stimuli so to minimize demand effect and experimental artifacts, randomizes participants to treatment and control conditions, and observes responses on a carefully chosen dependent variable. Not so for the "big data" necessary for natural language processing to give statistical

Much of the data used in the examples here, in common with many of the recent large scale analyses of human behavior online, are drawn from an enormous diversity of communi- 
ties with different norms, demographics, and changes over time. No explicit manipulation is applied to these systems. This means that researchers must combine robust statistical tools with an understanding of the idiosyncratic nature of the system to hand in order to generate reliable results.

In a demonstration of this, Boyce-Jacino and Chapman (2020) presented participants with a set of questions and answers taken from the online forum, Stack Exchange (SE). SE data is accompanied by rich metadata such as a rating of answer "quality"; each answer posted on the site receives "upvotes" from users who support that answer, and "downvotes" from those who view it as low quality.

For each SE question-answer pair, the authors solicited ratings on a variety of dimensions, including how relatable, specific, and polite the answers were. They find that the strongest predictors of the number of votes the answer received on the website are not those features that capture relatability or politeness. Rather, in that system, votes seemed to be reflecting solely perceptions of how specific and accurate the answers were. While this is, potentially, a victory for the site administrators, who developed a set of online norms and tools to encourage exactly this practice, it is a danger for the naieve psychologist who studies the system from the outside.

The work highlights the dangers of taking metadata at face value. If one wishes to use the "upvotes" of comments in discussion forums, or "likes" on a Twitter or Facebook post, to study the nature of question-asking, then it is important to understand what information is encoded by those actions. It is one thing to predict the votes given to a Stack Exchange post, but another to understand something deeper about the psychology of that action. People on SE who answer questions may want to provide relatable answers, and the question askers may appreciate it; it just so happens that SE's voting system is set up in a way to hide this effect.

The second set of challenges facing researchers in this domain arises from the computational tools themselves: are the chosen NLP tools appropriate for the task at hand? In a broad sense, the core of this question is to figure out whether or not NLP methods are able to answer cognitive questions about the nature of inquiry.

This chapter began with a discussion of how asking questions of other people requires a deeply intertwined process of social inference; a process which itself requires an interaction of individual cognitive processes and complex social reasoning. It then proceeded to offer distributional semantic models as a way to gain new insights into the process. These models are only useful, however, if they actually capture real-world semantics. If instead of being psychologically realistic models, they are simply engineering tools, any downstream claims about cognitive processing of difficulty or theory of mind, are perhaps less meaningful.

There are, however, several reasons to believe that vector space models are more than just useful engineering tools; for a comprehensive review of empirical and theoretical arguments, see Günther, Rinaldi, \& Marelli, 2019. Recall that models such as word2vec are founded on the hypothesis that words with similar meanings occur in similar contexts. They fundamentally argue that simple distributional statistics are a powerful source of information and are enough to define meaningful linguistic categories.

Supporting this claim, recent research has shown that distributional statistics are sufficient to facilitate novel concept acquisition (e.g., Ouyang, Boroditsky, \& Frank, 2017) and predict response latency in semantic priming experiments (e.g., Jones, Kintsch, \& Mewhort, 
2006; see also Günther et al., 2019). Within domains of higher order cognition, such as decision making (e.g., Bhatia, 2019) and moral judgments (e.g., Sagi \& Dehghani, 2014), vector space models also seem to capture preferences, reasoning fallacies, and judgments.

On an abstract level then, vector space models based on distributional semantics capture human semantic processing. To go beyond being simply useful for prediction, they must have inherent meaning: these models should reveal something about cognition and representation. Hollis and Westbury (2016) provide some initial evidence for this, by performing a principal components analysis on the 300-dimensional vectors learned by word2vec and show that the embedding model organizes words along dimensions long standard in psycholinguistic research, such as affective and lexical dimensions. The authors note that their findings do not necessarily imply that word2vec is a psychologically plausible model of semantics. Their findings do, however, suggest that such models learn and represent words in ways that mirror organization of words in human lexical semantics.

\section{Conclusion}

The last decade has led to an explosion of tools that make it possible to track rich features of language use on scales well beyond what can be done by traditional methods. There is every reason to believe that these tools will only become more accurate as the years go by.

This alone is not enough, however, to advance the science of psychology. We have yet to tie the outputs of NLP models, trained on text alone, to more basic cognitive processes in a satisfying fashion. Construct validity matters, just as much as it always has.

The ability to study behavior in the wild also brings with it new challenges. While laboratory studies can, through well-chosen stimuli, isolate one or another aspect of the task, behavior in the real world tends to mix everything together, in a balance that changes from dataset to dataset.

This requires that we develop theories as "big" as the datasets themselves. Their frameworks need to be flexible enough to address both the information search implicit in questionasking, as well as the larger, psychological features of cooperation and interaction. We have presented two examples from our own work - the decomposition of search into opacity and obscurity, and the five-feature Bayes cloud approach-but there is much more to do.

\section{Acknowledgments}

This research was funded by an National Science Foundation Doctoral Dissertation Research Grant in DRMS (SES-1948887) awarded to CB-J.

\section{References}

Alberti, C., Lee, K., \& Collins, M. (2019). A bert baseline for the natural questions. arXiv preprint arXiv:1901.08634. 
Baron-Cohen, S. (1991). Precursors to a theory of mind: Understanding attention in others. Natural theories of mind: Evolution, development and simulation of everyday mindreading, 1, 233-251.

Baroni, M., Dinu, G., \& Kruszewski, G. (2014). Don't count, predict! a systematic comparison of context-counting vs. context-predicting semantic vectors. In Proceedings of the 52nd annual meeting of the association for computational linguistics (volume 1: Long papers) (pp. 238-247).

Bhatia, S. (2019). Semantic processes in preferential decision making. Journal of Experimental Psychology: Learning, Memory, and Cognition, 45(4), 627.

Bhatia, S., \& Olivola, C. (2018). Data-driven computational brand perception. ACR North American Advances.

Bojanowski, P., Grave, E., Joulin, A., \& Mikolov, T. (2016). Enriching word vectors with subword information. arXiv preprint arXiv:160\%.04606.

Boyce-Jacino, C., \& Chapman, G. (2020). Preferences for answers. (In prep.)

Boyce-Jacino, C., \& DeDeo, S. (2019). The dynamics of human inquiry. In Society for philosophy and psychology.

Boyce-Jacino, C., \& DeDeo, S. (2020). Opacity, obscurity, and the geometry of questionasking. Cognition, 196, 104071. doi: https://doi.org/10.1016/j.cognition.2019.104071

Branigan, H. P., Pickering, M. J., \& Cleland, A. A. (2000). Syntactic co-ordination in dialogue. Cognition, $75(2)$, B13-B25.

Chali, Y., \& Hasan, S. A. (2015). Towards topic-to-question generation. Computational Linguistics, 41(1), 1-20.

Coenen, A., Nelson, J. D., \& Gureckis, T. M. (2019). Asking the right questions about the psychology of human inquiry: Nine open challenges. Psychonomic Bulletin \& Review, 26 (5), 1548-1587.

Cohen, A., \& Lake, B. M. (2016). Searching large hypothesis spaces by asking questions. In Cogsci.

Devlin, J., Chang, M.-W., Lee, K., \& Toutanova, K. (2018). BERT: Pre-training of deep bidirectional transformers for language understanding.

Firth, J. R. (1957). A synopsis of linguistic theory, 1930-1955. Studies in linguistic analysis.

Garrod, S., \& Anderson, A. (1987). Saying what you mean in dialogue: A study in conceptual and semantic co-ordination. Cognition, 27(2), 181-218.

Garrod, S., \& Clark, A. (1993). The development of dialogue co-ordination skills in schoolchildren. Language and Cognitive Processes, 8(1), 101-126.

Griffiths, T. L., Chater, N., Kemp, C., Perfors, A., \& Tenenbaum, J. B. (2010). Probabilistic models of cognition: Exploring representations and inductive biases. Trends in cognitive sciences, 14 (8), 357-364.

Günther, F., Rinaldi, L., \& Marelli, M. (2019). Vector-space models of semantic representation from a cognitive perspective: A discussion of common misconceptions. Perspectives on Psychological Science, 14(6), 1006-1033.

Harris, Z. S. (1954). Distributional structure. Word, 10(2-3), 146-162.

Hawkins, R., \& Goodman, N. D. (2020, Apr). Questions and answers in dialogue. OSF. Retrieved from osf.io/p2gyr

Hollis, G., \& Westbury, C. (2016). The principals of meaning: Extracting semantic dimensions from co-occurrence models of semantics. Psychonomic bulletin $\&$ review, 23(6), 
1744-1756.

Jones, M. N., Kintsch, W., \& Mewhort, D. J. (2006). High-dimensional semantic space accounts of priming. Journal of memory and language, 55(4), 534-552.

Kennedy, B., Jin, X., Davani, A. M., Dehghani, M., \& Ren, X. (2020). Contextualizing hate speech classifiers with post-hoc explanation. arXiv preprint arXiv:2005.02439.

Leslie, A. M. (1987). Pretense and representation: The origins of" theory of mind.". Psychological review, $94(4), 412$.

Levelt, W. J., \& Kelter, S. (1982). Surface form and memory in question answering. Cognitive psychology, 14(1), 78-106.

Lewis, M., \& Lupyan, G. (2019). What are we learning from language? associations between gender biases and distributional semantics in 25 languages.

Mandera, P., Keuleers, E., \& Brysbaert, M. (2017). Explaining human performance in psycholinguistic tasks with models of semantic similarity based on prediction and counting: A review and empirical validation. Journal of Memory and Language, 92, 57-78.

Mikolov, T., Sutskever, I., Chen, K., Corrado, G. S., \& Dean, J. (2013). Distributed representations of words and phrases and their compositionality. In Advances in neural information processing systems (pp. 3111-3119).

Moore, R., Mueller, B., Kaminski, J., \& Tomasello, M. (2015). Two-year-old children but not domestic dogs understand communicative intentions without language, gestures, or gaze. Developmental Science, 18(2), 232-242.

Mostafazadeh, N., Misra, I., Devlin, J., Mitchell, M., He, X., \& Vanderwende, L. (2016). Generating natural questions about an image. arXiv preprint arXiv:1603.06059.

Nelson, J. D. (2005). Finding useful questions: on bayesian diagnosticity, probability, impact, and information gain. Psychological review, 112(4), 979.

Nickel, M., \& Kiela, D. (2017). Poincaré embeddings for learning hierarchical representations. In Advances in neural information processing systems (pp. 6338-6347).

Oaksford, M., Chater, N., et al. (2007). Bayesian rationality: The probabilistic approach to human reasoning. Oxford University Press.

Onishi, K. H., \& Baillargeon, R. (2005). Do 15-month-old infants understand false beliefs? science, 308(5719), 255-258.

Ouyang, L., Boroditsky, L., \& Frank, M. C. (2017). Semantic coherence facilitates distributional learning. Cognitive science, 41, 855-884.

Pennington, J., Socher, R., \& Manning, C. D. (2014). GloVe: Global vectors for word representation. In Proceedings of the 2014 conference on empirical methods in natural language processing (EMNLP) (pp. 1532-1543).

Pereira, F., Lou, B., Pritchett, B., Ritter, S., Gershman, S. J., Kanwisher, N., .. Fedorenko, E. (2018). Toward a universal decoder of linguistic meaning from brain activation. Nature Communications, 9(1), 1-13.

Perner, J. (1991). Understanding the representational mind. The MIT Press.

Potamias, R. A., Siolas, G., \& Stafylopatis, A.-G. (2019). A transformer-based approach to irony and sarcasm detection. arXiv preprint arXiv:1911.10401.

Rajpurkar, P., Zhang, J., Lopyrev, K., \& Liang, P. (2016). Squad: 100,000+ questions for machine comprehension of text.

Rothe, A., Lake, B. M., \& Gureckis, T. (2017). Question asking as program generation. In Advances in neural information processing systems (pp. 1046-1055). 
Rothe, A., Lake, B. M., \& Gureckis, T. M. (2016). Asking and evaluating natural language questions. In Cogsci.

Sagi, E., \& Dehghani, M. (2014). Moral rhetoric in twitter: A case study of the US Federal Shutdown of 2013. In Proceedings of the annual meeting of the cognitive science society (Vol. 36).

Scholl, B. J., \& Gao, T. (2013). Perceiving animacy and intentionality: Visual processing or higher-level judgment. Social perception: Detection and interpretation of animacy, agency, and intention, 4629.

Searle, J. R. (1969). Speech acts: An essay in the philosophy of language (Vol. 626). Cambridge university press.

Steyvers, M., \& Tenenbaum, J. B. (2005). The large-scale structure of semantic networks: Statistical analyses and a model of semantic growth. Cognitive science, 29(1), 41-78.

Tenenbaum, J. B., Griffiths, T. L., \& Kemp, C. (2006). Theory-based bayesian models of inductive learning and reasoning. Trends in cognitive sciences, 10(7), 309-318.

Tenney, I., Xia, P., Chen, B., Wang, A., Poliak, A., McCoy, R. T., .. Pavlick, E. (2019). What do you learn from context? probing for sentence structure in contextualized word representations. CoRR, abs/1905.06316. Retrieved from http://arxiv.org/ abs/1905.06316

Tomasello, M., Call, J., \& Gluckman, A. (1997). Comprehension of novel communicative signs by apes and human children. Child development, 68(6), 1067-1080.

Ullman, T., Baker, C., Macindoe, O., Evans, O., Goodman, N., \& Tenenbaum, J. B. (2009). Help or hinder: Bayesian models of social goal inference. In Advances in neural information processing systems (pp. 1874-1882).

Vaswani, A., Shazeer, N., Parmar, N., Uszkoreit, J., Jones, L., Gomez, A. N., .. Polosukhin, I. (2017). Attention is all you need. In I. Guyon et al. (Eds.), Advances in neural information processing systems 30 (pp. 5998-6008). Curran Associates, Inc. Retrieved from http://papers.nips.cc/paper/7181-attention-is-all-you-need.pdf

Wang, Z., \& Lake, B. M. (2019). Modeling question asking using neural program generation. arXiv preprint arXiv:190\%.09899.

Wang, Z., Ng, P., Ma, X., Nallapati, R., \& Xiang, B. (2019). Multi-passage bert: A globally normalized bert model for open-domain question answering. arXiv preprint arXiv:1908.08167.

Wimmer, H., \& Perner, J. (1983). Beliefs about beliefs: Representation and constraining function of wrong beliefs in young children's understanding of deception. Cognition, $13(1), 103-128$.

Xu, H., Liu, B., Shu, L., \& Yu, P. S. (2019). Bert post-training for review reading comprehension and aspect-based sentiment analysis. arXiv preprint arXiv:1904.02232.

Yu, Y., Bonawitz, E., \& Shafto, P. (2019). Pedagogical questions in parent-child conversations. Child development, 90(1), 147-161. 